\title{
Protein-Protein Interaction in Purple Membrane
}

\author{
Maikel C. Rheinstädter, ${ }^{1,2,4 *}$ Karin Schmalzl, ${ }^{3}$ Kathleen Wood, ${ }^{4,5, \dagger}$ and Dieter Strauch ${ }^{6}$ \\ ${ }^{1}$ Department of Physics and Astronomy, McMaster University, Hamilton, Ontario, L8S 4M1, Canada \\ ${ }^{2}$ Canadian Neutron Beam Centre, Chalk River, Ontario, KOJ 1JO, Canada \\ ${ }^{3}$ Institut für Festkörperforschung, Forschungszentrum Jülich GmbH, JCNS at ILL, F-38042 Grenoble, France \\ ${ }^{4}$ Institut Laue-Langevin, F-38042 Grenoble Cedex 9, France \\ ${ }^{5}$ Institut de Biologie Structurale Jean Pierre Ebel CEA-CNRS-UJF, F-38027 Grenoble, France \\ ${ }^{6}$ Theoretische Physik, Universität Regensburg, D-93040 Regensburg, Germany
}

(Received 7 April 2009; published 18 September 2009)

\begin{abstract}
We present experimental evidence for a long-range protein-protein interaction in purple membrane (PM). The interprotein dynamics were quantified by measuring the spectrum of the acoustic phonons in the $2 \mathrm{D}$ bacteriorhodopsin (BR) protein lattice using inelastic neutron scattering. Phonon energies of about $1 \mathrm{meV}$ were determined. The data are compared to an analytical model, and the effective spring constant for the interaction between neighboring protein trimers are determined to be $k=53 \mathrm{~N} / \mathrm{m}$. Additional, optical-like excitations at $0.45 \mathrm{meV}$ were found and assigned to intraprotein dynamics between neighboring BR monomers.
\end{abstract}

DOI: 10.1103/PhysRevLett.103.128104

PACS numbers: 87.15.km, 83.85.Hf, 87.16.dj

The high protein concentration in biological membranes may lead to long-range protein-protein interactions, which were speculated some time ago [1]. Motions in proteins occur on various length and time scales [2,3], and the functional behavior of membrane proteins is likely to depend on the lipid bilayer composition and physical properties, such as hydrophobic thickness and elastic moduli. How the variety of inter- and intraprotein motions, occurring over different time and length scales, interact to result in a functioning biological system remains an open field for those working at the interface of physics and biology. The dynamical coupling between proteins, i.e., cooperative protein dynamics, may be important for the understanding of macromolecular function in a cellular context because it can lead to an effective interprotein communication. Recently, interprotein motions in a carboxymyoglobin protein crystal were reported from a molecular dynamics simulation $[4,5]$. Experimentally, phononlike excitations of proteins in hydrated protein powder were reported [6]. Here, we report collective interprotein excitations in a biological membrane, the purple membrane (PM).

PM occurs naturally in the form of a two-dimensional crystal, consisting of $75 \%$ (wt/wt) of a single protein, bacteriorhodopsin (BR), that functions as a light-activated proton pump, and 25\% various lipid species (mostly phospho- and glycolipids) [7]. BR is a proton transporting membrane protein, formed of seven transmembrane alpha helices arranged around the photosensitive retinal molecule. The protein in the lipid matrix is organized in trimers that form a highly ordered 2D hexagonal lattice with lattice parameter $a \approx 62 \AA$. The structure of PM is well established by electron microscopy, neutron and x-ray diffraction experiments as reviewed for instance in [7-12]. Here we present the unprecedented determination of collective protein-protein dynamics, i.e., acoustic phonons, in the 2D protein lattice in PM using coherent inelastic neutron scattering. The results allow the determination of the effective coupling constant by comparison to an analytical model.

The experiments were performed on the IN12 coldtriple-axis spectrometer at the Institut Laue-Langevin (ILL, Grenoble, France). IN12 turned out to be highly suited for elastic and inelastic investigations in oriented biological samples because of its flexibility, good energy resolution, and extremely low background. It allows the measurement of diffraction and inelastic scattering in the same run without changing the setup, which is crucial to assign dynamical modes to structural properties and molecular components. IN12 was equipped with a vacuum box to avoid air scattering at small scattering angles and with vertically focusing monochromator and analyzer to increase the neutron flux at the sample position. There was no horizontal focusing, but the beam was collimated to $40^{\prime}$ - monochromator - $30^{\prime}$ - sample - $30^{\prime}$ - analyzer$60^{\prime}$ - detector. All scans were done in the $W$ configuration with fixed $k_{f}=1.25 \AA^{-1}$ resulting in a $q$ resolution of $\Delta q=0.005 \AA^{-1}$, and an energy resolution of $\Delta \hbar \omega=$ $25 \mu \mathrm{eV}$.

Deuterated PM was produced and hydrated by $\mathrm{H}_{2} \mathrm{O}$ in order to suppress the contribution of the membrane hydration water to the phonon spectrum in the coherent inelastic neutron scattering experiments. Because of the minuteness of the coherent inelastically scattered signals, the preparation of appropriate samples and experimental setups is challenging in this type of experiments. We used a completely deuterated PM to enhance the collective proteinprotein excitations over other contributions to the inelastic scattering cross section. $200 \mathrm{mgs}$ of deuterated PM sus- 

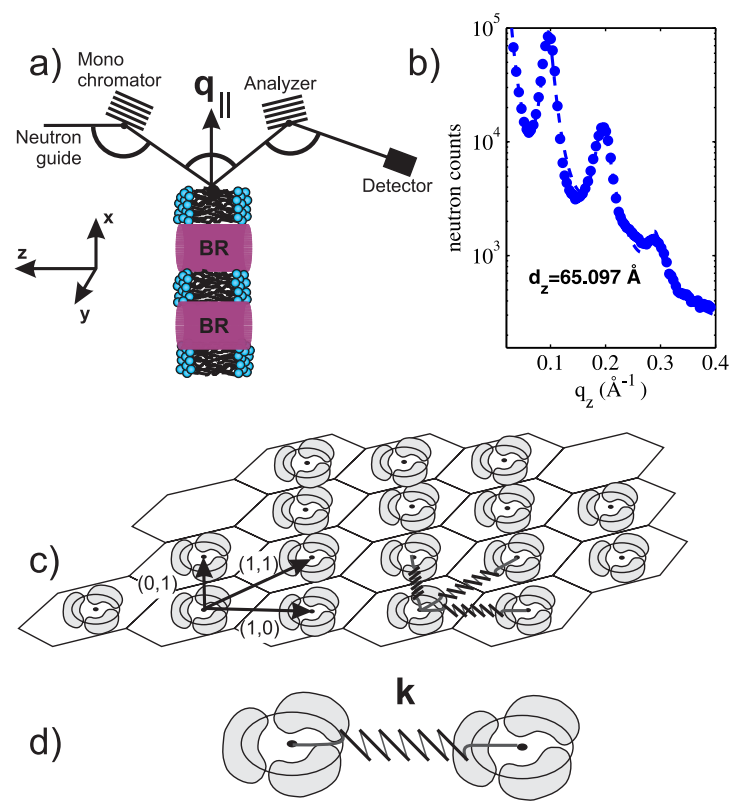

FIG. 1 (color online). (a) Sketch of the triple-axis scattering geometry. $q_{\|}$is the in-plane component of the scattering vector Q. (b) Reflectivity curve measured at $T=30^{\circ} \mathrm{C}$. The dotted line is a fit of Lorentzian peak profiles including a $q^{-4}$ term. (c) BR trimers are arranged on a hexagonal lattice of lattice constant $a \approx 62 \AA$. (d) The interaction between the protein trimers is depicted as springs with effective spring constant $k$.

pended in $\mathrm{H}_{2} \mathrm{O}$ was centrifuged down and the obtained pellet spread onto a $40 \times 30 \mathrm{~mm}$ aluminum sample holder. This was then partially dried to $0.5 \mathrm{~g}$ water per gram of membrane over silica gel in a desiccator. The PM patches naturally align along the surface of the sample holder as they dry. The silica gel was then replaced by water, and the sample left to hydrate to a lamellar spacing of $d_{z}=65 \AA$ at $303 \mathrm{~K}\left(30^{\circ} \mathrm{C}\right)$.

A sketch of the scattering geometry is shown in Fig. 1(a). The experiments were carried out on PM stacks, i.e., PM samples with a regular repeat distance $d_{z}$. The mosaicity of the sample (the distribution of normal vectors with respect to the substrate) was checked by rocking scans to about $17^{\circ}$. Figure 1 (b) shows a reflectivity curve measured at $T=30^{\circ} \mathrm{C}$. From the three well developed Bragg peaks, the lamellar spacing $d_{z}$ was determined to be $d_{z}=$ $65.1 \AA$, corresponding to an average inter membrane water layer of $16 \AA$, since the thickness of a dry PM fragment is $49 \AA$. For the inelastic scans, $\mathbf{Q}$ was placed in the plane of the membranes $\left(q_{\|}\right)$.

The in-plane diffraction pattern of the 2D hexagonal protein lattice was measured and is shown in Fig. 2. Although the normal vectors of the membranes are well aligned with respect to the substrate, the $(x, y)$ orientation of the membrane layers is statistical and the signal a superposition of the different domains (powder average). All reflections can be indexed by a hexagonal unit cell with a lattice parameter of $61.78 \pm 0.73 \AA$. Correlations and motions in membranes are often well separated in recip-

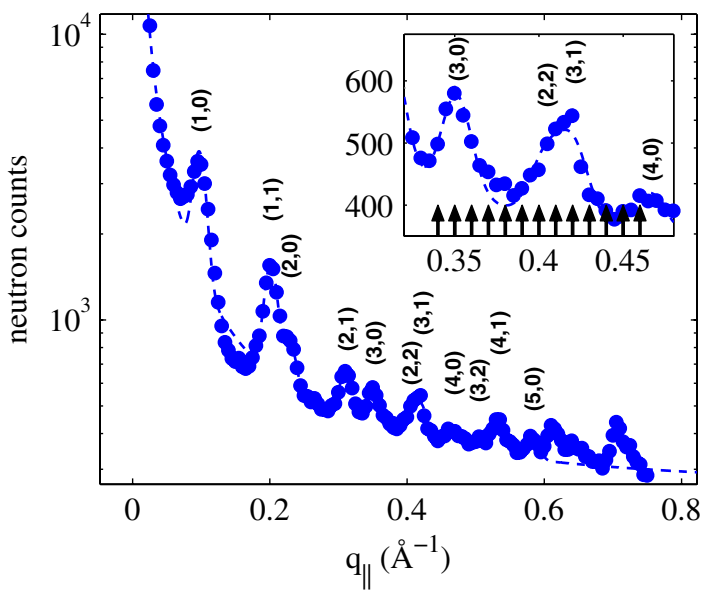

FIG. 2 (color online). Diffraction pattern of the 2D protein lattice from $q_{\|}=0$ to $0.75 \AA^{-1}$. The reflections can be indexed by a hexagonal unit cell with a lattice parameter of $61.78 \pm$ $0.73 \AA$. The dashed line is a fit of the theoretical peak pattern to the data. The inset shows the third Brillouin zone in magnification. The arrows mark the positions of inelastic scans at constant $q_{\|}$values.

rocal space because of the largely different length and time scales involved. The prominent distances in PM, such as lipid-lipid and BR-BR monomers and trimers for instance lead to spatially well-separated signals. The same holds for the different time scales involved from the picosecond (molecular reorientations) to the nano- or microsecond (membrane undulations, large protein motions). The use of oriented samples further allows the separation of correlations in the plane of the membranes, and perpendicular to the bilayers. Dynamics between different protein trimers is expected to be dominant where the 2D BR diffraction pattern is observed, i.e., in a $q_{\|}$range of about $0.1 \AA^{-1}$ to $0.6 \AA^{-1}$. Because elastic and inelastic scattering at small momentum transfers was dominated by the rather strong $(1,0)$ and $(1,1)$ reflections, systematic inelastic scans were taken at $q_{\|}$values in the third Brillouin zone of the 2D pattern, between $0.34 \AA^{-1}$ and $0.46 \AA^{-1}$. The inset in Fig. 2 shows the Bragg peaks in the third Brillouin zone in magnification and marks the positions of inelastic scans at constant $q_{\|}$values. Figure 3 depicts constant- $q_{\|}$scans at selected values between $q_{\|}=0.35 \AA^{-1}$ and $q_{\|}=$ $0.45 \AA^{-1}$. The total inelastic signal consists of a Gaussian central peak due to instrumental resolution, a quasielastic broadening, which is described by a Lorentzian peak shape, and pairs of excitations, described by damped harmonic oscillator peak profiles. Because of the pronounced and symmetric inelastic signals, we are sure that the observed peaks are not spurious effects. The quasielastic signal most likely stems from coherent and incoherent diffusive motions.

The excitation spectrum of the $2 \mathrm{D}$ protein lattice was modeled analytically. The protein trimers were taken as the centers of a primitive hexagonal lattice with lattice constant $a=62 \AA$, and the spectrum of the acoustic phonons 


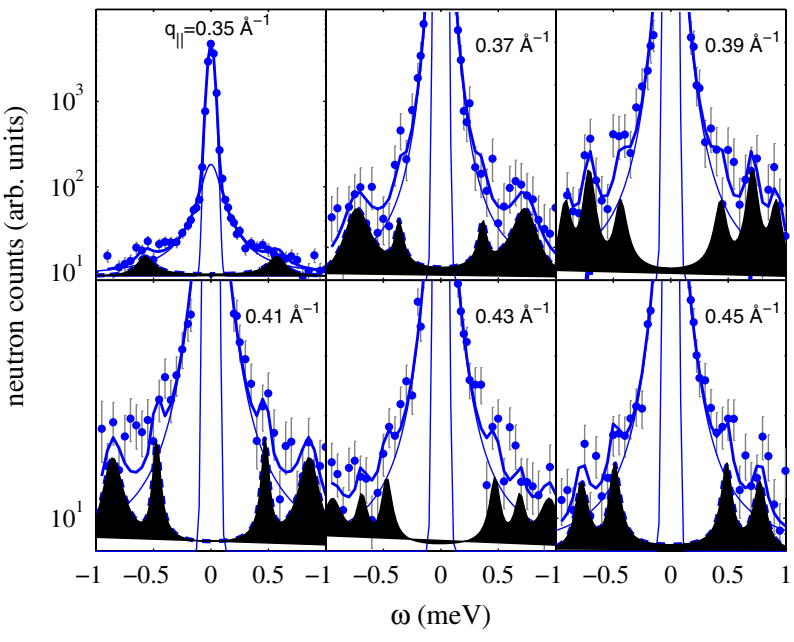

FIG. 3 (color online). Energy scans at $q_{\|}$values of $q_{\|}=0.35$, $0.37,0.39,0.41,0.43$, and $0.45 \AA^{-1}$. The total inelastic signal consists of a central peak (Gaussian), a Lorentzian quasielastic contribution, and the excitations, fitted using damped harmonic oscillator peak profiles.

was calculated. The model is depicted in Fig. 1(c). The basic hexagonal translations are marked by arrows. The interaction between the protein trimers is contained in springs with an effective (longitudinal) spring constant $k$ [Fig. 1(d)]. The calculated longitudinal spectrum $C_{l}(q, \omega)$, defined by $C_{l}(q, \omega)=\left(\omega^{2} / q^{2}\right) S(q, \omega)$, is shown in Fig. 4. The statistical average in the plane of the membrane leads to a superposition of the different phonon branches, which start and end in the hexagonal Bragg peaks (at $\hbar \omega=0$ ). The positions of excitations, as determined from the fits in Fig. 3, are marked by the data points. The absolute phonon energies can not be determined from the model, but depend

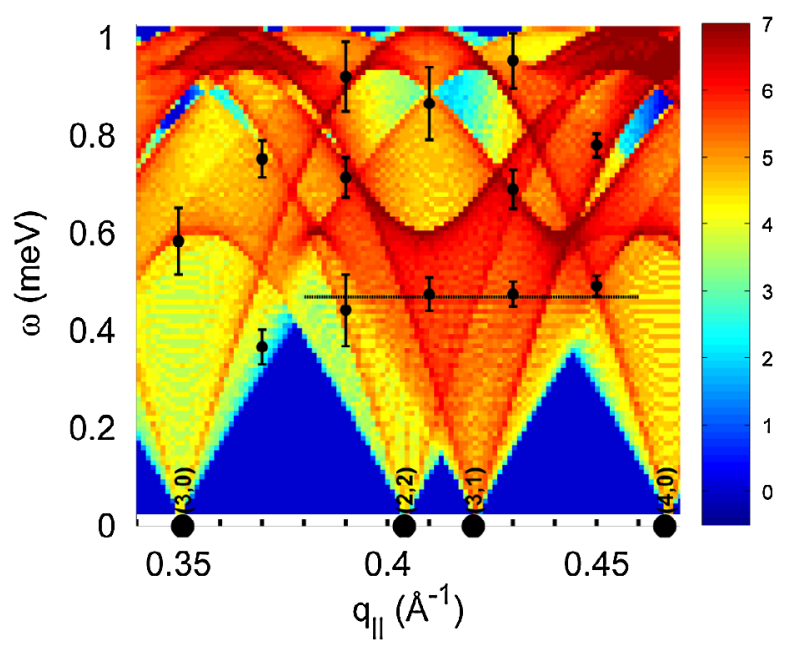

FIG. 4 (color online). Calculated excitation spectrum $C_{l}(q, \omega)$ in the range of the experimental data. Data points mark the positions of excitations, as determined from the fits in Fig. 3. The errorbars give the uncertainty in determining the peak position. The horizontal line at $\omega=0.45 \mathrm{meV}$ marks the position of a possible optical phonon mode, not included in the calculations. on the coupling constant $k$. So the energy of the phonon spectrum in Fig. 4 was fitted to best match the experiment. The experimentally determined excitation energies occur at the intersections of the inelastic scans with the calculated phonon branches and well reproduce the features of the theoretical excitation spectrum (within the given errors). Note that because the proteins trimers were treated as point masses with an effective mass of $M_{\text {tr }}$, the calculations do not include any contributions from intramonomer or intratrimer dynamics, i.e., possible optical modes. The excitations observed in the experiment at $q_{\|}$values around $0.42 \AA^{-1}$ (corresponding to a length scale of about $15 \AA$ ) and energy value of $0.45 \mathrm{meV}$ (marked by the dashed line in Fig. 4), have no equivalent in the theoretical spectrum and possibly stem from optical phonon branches. Because the distance agrees well with the monomer-monomer distance in the protein trimer this mode can tentatively be assigned to optical-like monomer dynamics (the corresponding form factor is likely to be maximum at the corresponding nearest neighbor distance).

The commonly assumed interaction mechanism between inclusions in membranes is a lipid-mediated interaction due to local distortions of the lipid bilayer [13-17], with a strong dependence on the bilayer properties, in particular, elastic properties. The PM may, however, be a special case because there are very few lipids between neighboring BR proteins [18]. While the nature of the interaction still will be mainly elastic, it is not likely to be purely lipid mediated but for the most part a direct protein-protein interaction. The strength of the interaction can be determined from the data in Fig. 4. The energy of the zone-boundary phonon at the $M$ point of the hexagonal Brillouin zone (for instance at a $q_{\|}$value of $0.35 \AA^{-1}$ ) relates to the coupling constant by $M_{\mathrm{tr}} \omega^{2}=6 k$. Because the energy is determined to $\hbar \omega=1.02 \mathrm{meV}$, the effective protein-protein spring constant $k$ is calculated to $k=$ $53 \mathrm{~N} / \mathrm{m}$ [19]. The amplitude of this mode of vibration can be estimated from the equipartition theorem to $\sqrt{\left\langle x^{2}\right\rangle}=\sqrt{k_{B} T / k}=0.1 \AA$, and the interaction force between two neighboring trimers to $F=k \sqrt{\left\langle x^{2}\right\rangle}=0.5 \mathrm{nN}$. Using the same approach, the spring constant for graphite for comparison is calculated to $27000 \mathrm{~N} / \mathrm{m}$ for the inplane interaction, and $3.5 \mathrm{~N} / \mathrm{m}$ for out-of-plane interactions. The force constant that we measure in PM is 1-2 orders of magnitude larger than the effective van der Waals force constant in graphite, but 2-3 orders of magnitude weaker than a $\mathrm{C}-\mathrm{C}$ bond.

The "softness" of BR proteins in PM was probed by elastic incoherent neutron scattering $[8,20]$, and force constants of $0.1 \mathrm{~N} / \mathrm{m}$ were reported. The shear modulus in stacks of PM was determined by $\mathrm{x}$-ray reflectivity to $\mu=$ $0.02 \mathrm{~N} / \mathrm{m}$ [12]. The corresponding amplitudes of vibration were 2.2 respective $3.8 \AA$. These techniques were mainly sensitive to diffusion of proteins (and lipids), and the force and time constants involved are determined by local friction and restoring forces. The corresponding force con- 
stants are about 2 orders of magnitude smaller than the one reported in this study. Energies of collective, phononlike excitations were also reported for proteins in a protein crystal (from computer simulations $[4,5]$ ) and hydrated protein powder (from inelastic x-ray scattering [6]), and energies of about $1 \mathrm{meV}$ for inter-, and even $10 \mathrm{meV}$ for intraprotein motions were found. The reported energies cannot be quantitatively compared to the excitations in this study because the systems are distinctly different. However, it seems that the energies found for protein interactions are consistently higher than the force constants from experiments, which probe the local energy landscapes. The amplitudes of diffusive, self-correlated motions are therefore about one magnitude larger than those of collective, pair correlated motions. "Motional coherence", i.e., a structurally coherent state, was reported recently in fluid phospholipid membranes [21]. So while for a long time motions in biological materials were considered as thermally activated vibrations or librations in local potentials, at least part of the fluctuation spectrum stems from interactions. The future challenge is to understand the impact of collective molecular motions in membranes and proteins on biological function.

The protein coupling reported here may be relevant for the photo cycle in PM. It was reported [22] that the BR proteins undergo structural changes during the photo cycle, involving displacements of up to $1.7 \AA$. Because of the elastic coupling of the BR proteins, those distortions can propagate to neighboring proteins. On the microscopic level, displacing or distorting a BR trimer by $1.7 \AA$ yields a force between neighboring trimers of $9 \mathrm{nN}$ (using the model presented here). It can therefore be speculated that there is a protein-protein communication during the photocycle in PM. So while thermal fluctuations are dominated by diffusive motions, collective motions may play a key role to establish dynamics-function relations in complex biological materials.

In conclusion we present experimental evidence for protein-protein interactions in purple membrane. The spectrum of the acoustic phonons in the 2D bacteriorhodopsin lattice was calculated and measured by inelastic neutron scattering. Phonon energies of about $1 \mathrm{meV}$ were found. An additional band at energies of about $0.45 \mathrm{meV}$ was tentatively assigned to optical phonon modes between the BR monomers. The effective spring constant for the interaction between protein trimers was determined from the acoustic phonon branches to $k=53 \mathrm{~N} / \mathrm{m}$. Future experiments will address collective intraprotein trimer and also intramonomer dynamics. To make a clear relationship to protein function, protein dynamics of activated proteins, i.e., the collective dynamics of proteins undergoing the photo cycle, will be studied [23]. While the protein concentration in PM is very high and the proteins are very close to a $2 \mathrm{D}$ crystal, it can be speculated that there is a protein coupling also in less dense membrane systems.
We thank Brigitte Kessler and Dieter Oesterhelt (MPI for Biochemistry) for providing the sample, Martin Weik (IBS) for help with sample preparation, Giuseppe Zaccai (ILL) for critical reading of the manuscript, and the Institut Laue-Langevin for the allocation of beam time.

*rheinstadter@mcmaster.ca

†Present address: Australian Nuclear Science \& Technology Organisation, Bragg Institute, Menai, Australia.

[1] Structure and Dynamics of Membranes, edited by R. Lipowsky and E. Sackmann, Handbook of Biological Physics Vol. 1 (Elsevier, Amsterdam, 1995).

[2] H. Frauenfelder, S. Sligar, and P. Wolynes, Science 254, 1598 (1991).

[3] P. Fenimore, H. Frauenfelder, B. McMahon, and R. Young, Proc. Natl. Acad. Sci. U.S.A. 101, 14408 (2004).

[4] V. Kurkal-Siebert, R. Agarwal, and J. C. Smith, Phys. Rev. Lett. 100, 138102 (2008).

[5] L. Meinhold, J.C. Smith, A. Kitao, and A. H. Zewail, Proc. Natl. Acad. Sci. U.S.A. 104, 17261 (2007).

[6] D. Liu, X.-Q. Chu, M. Lagi, Y. Zhang, E. Fratini, P. Baglioni, A. Alatas, A. Said, E. Alp, and S.-H. Chen, Phys. Rev. Lett. 101, 135501 (2008).

[7] U. Haupts, J. Tittor, and D. Oesterhelt, Annu. Rev. Biophys. Biomol. Struct. 28, 367 (1999).

[8] G. Zaccai, Biophys. Chem. 86, 249 (2000).

[9] R. Neutze, E. Pebay-Peyroula, K. Edman, A. Royant, J. Navarro, and E. Landau, Biochim. Biophys. Acta 1565, 144 (2002).

[10] J. Lanyi, Annu. Rev. Physiol. 66, 665 (2004).

[11] I. Koltover, T. Salditt, J.-L. Rigaud, and C. Safinya, Phys. Rev. Lett. 81, 2494 (1998).

[12] I. Koltover, J. Rädler, T. Salditt, and C. Safinya, Phys. Rev. Lett. 82, 3184 (1999).

[13] P. A. Kralchevsky, Adv. Biophys. 34, 25 (1997).

[14] K. Bohinc, V. Kralj-Iglič, and S. May, J. Chem. Phys. 119, 7435 (2003).

[15] P. Biscari and F. Bisi, Eur. Phys. J. E 7, 381 (2002).

[16] P. Lagüe, M. J. Zuckermann, and B. Roux, Biophys. J. 81, 276 (2001).

[17] N. Dan, P. Pincus, and S. Safran, Langmuir 9, 2768 (1993).

[18] J. Baudry, E. Tajkhorshid, F. Molnar, J. Phillips, and K. Schulten, J. Phys. Chem. 105, 905 (2001).

[19] Hunt et al., determined the molecular weight of a BR monomer was determined to $26.9 \mathrm{kDa}(1 \mathrm{~kg}=6.0221 \times$ $10^{26}$ dalton); J. F. Hunt, P. D. McCrea, G. Zaccaï, and D. M. Engelman, J. Mol. Biol. 273, 1004 (1997).

[20] G. Zaccai, Science 288, 1604 (2000).

[21] M. C. Rheinstädter, J. Das, E. J. Flenner, B. Brüning, T. Seydel, and I. Kosztin, Phys. Rev. Lett. 101, 248106 (2008).

[22] H. Luecke, B. Schobert, H.-T. Richter, J.-P. Cartailler, and J. K. Lanyi, Science 286, 255 (1999).

[23] J. Pieper, A. Buchsteiner, N. A. Dencher, R. E. Lechner, and T. Hauß, Phys. Rev. Lett. 100, 228103 (2008). 